\title{
Study on Decomposition of Multialkylated Cyclopentane Oil with Sulfur-Containing Additive on the Nascent Steel Surface
}

\author{
Renguo Lu, Shigeyuki Mori ${ }^{*}$, Hidetaka Nanao, Kimihiro Kobayashi and Ichiro Minami \\ Department of Chemical Engineering, Iwate University \\ 4-3-5 Ueda, Morioka, Iwate 020-8551, Japan \\ *Corresponding author: mori@iwate-u.ac.jp
}

( Manuscript received 14 August 2007; accepted 11 November 2007; published 30 November 2007 )

\begin{abstract}
Effect of di-tert-dodecyl disulfide as an additive on decomposition of MAC oil on the nascent surface of bearing steel 52100 was investigated. After the removal of surface contamination layer, active sites were generated, on which tribochemical decomposition of MAC oil occurred. The additive prolonged the induction period of the decomposition. Hydrogen and gaseous hydrocarbons desorbed as tribochemical reaction products. Desorption rate of hydrogen and gaseous hydrocarbons increased proportionally with sliding velocity and linearly with the cube root of load. XPS analysis revealed tribochemical reaction between additive and nascent surface occurred, which induced formation of iron sulfide and decrease of activity of nascent surface. As a result, decomposition of MAC oil decreased correspondingly.
\end{abstract}

Keywords: nascent surface, tribochemical reaction, lubricant decomposition, organic sulfide

\section{Introduction}

Synthetic multialkylated cyclopentanes (MACs) have been accepted widely as a base oil in advanced fields of technology, such as spacecraft mechanisms ${ }^{1-3)}$. MACs show low vapor pressure, high thermal and chemical stability ${ }^{4}$. Under friction conditions and in contact with metals, nascent surface with many active sites will be generated easily by the removal of surface layers such as metal oxides and organic contaminations. MACs partially decompose, resulting in the formation of low molecular weight products, such as $\mathrm{H}_{2}^{+}, \mathrm{CH}_{3}^{+}$, $\mathrm{C}_{2} \mathrm{H}_{5}{ }^{+2,5)}$. The produced hydrogen interacts with metal leading to hydrogen embrittlement ${ }^{6)}$. Contamination of low molecular weight products will be harmful to the vacuum systems. Lubricant loss leads to oil film disruption and surface damage.

This paper presents a part of the larger study on MACs tribochemistry, focusing the mechanism of MACs decomposition with the additive of di-tert-dodecyl disulfide on the nascent surface of steel.

\section{Experimental details}

Schematic diagram of the test apparatus used in this study is illustrated in Figure 1, and described in detail elsewhere ${ }^{5,7-9)}$. A ball-on-disk type sliding tester was installed in a high vacuum chamber with a base pressure less than $2 \times 10^{-5} \mathrm{~Pa}$. A thin lubricant film was formed on the disk surface. During the rubbing test, the pressure in the vacuum chamber was measured by a Bayard-Alpert ion gauge, and intensities of the molecular and fragment ions coming from reaction products were monitored by a quadrupole mass spectrometer (Q-MS). All the tests were carried out on the same friction track by using the same ball at room temperature. Load and sliding velocity were changed from 2 to $16 \mathrm{~N}$, and from 0.02 to $0.06 \mathrm{~m} / \mathrm{s}$ respectively.

Figure 2 shows a typical chart from a mass spectrometer. For the first 1 minute, the sliding tester was left at rest and it was confirmed that the baselines of ion intensities were stable. For the next 2 minutes, the sliding test was carried out and the intensity changes were recorded. For the last 2 minutes, the sliding tester was left at rest and the stability of the baselines was confirmed again.

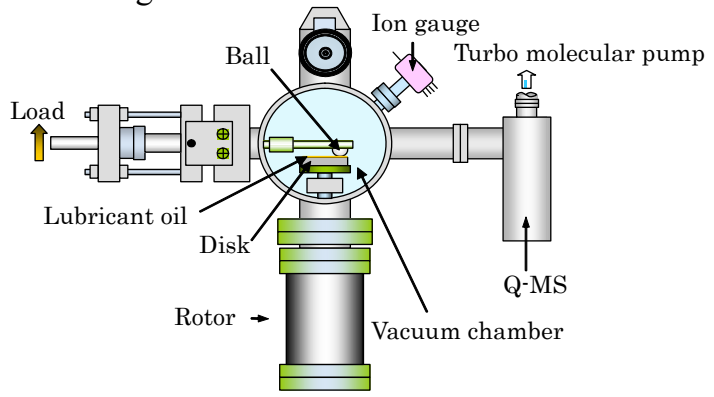

Fig.1 Schematic diagram of the test apparatus for investigation of chemical nature of nascent surface 


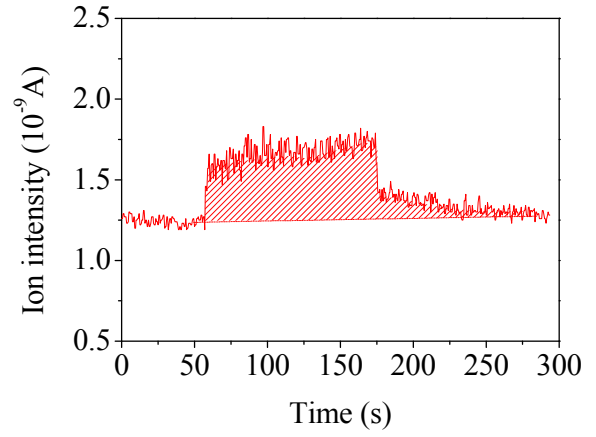

Fig.2 A typical chart of mass spectrometer $(\mathrm{m} / \mathrm{e}=2)$

In this study, ball and disk specimens were made of bearing steel 52100 . The ball was $6.35 \mathrm{~mm}$ in diameter. The disk was $24 \mathrm{~mm}$ in diameter and $7 \mathrm{~mm}$ in thickness. The disk was polished to an average roughness of $\mathrm{Ra}$ $0.02 \mu \mathrm{m}$. The specimens were successively cleaned for 10 min in an ultrasonic bath with petroleum ether and hexane, respectively before sliding test.

The oil used in these experiments was synthetic multialkylated cyclopentane, 1, 2, 4-Tris (2-Octyl-1-Dodecyl) Cyclopentane, produced by Nye Lubricants, Inc. and was used without further processing. Main physical properties of the oil are shown in Table 1. MAC oil mixed with $1 \mathrm{wt} \%$ of di-tert-dodecyl disulfide was examined in this study.

Table 1 Properties of Sample Oil

\begin{tabular}{l|l|l}
\hline Chemical structure & $\mathrm{R}^{\mathrm{R}}$ & \multicolumn{2}{|c}{$\mathrm{C}_{8} \mathrm{H}_{17}$} \\
\hline Kinematic & $\mathrm{R}$ & $\mathrm{R}:-\mathrm{CH}_{2}-\mathrm{CH}-\mathrm{C}_{10} \mathrm{H}_{21}$ \\
Viscosity (cSt) & $100^{\circ} \mathrm{C}$ & 14.6 \\
\cline { 2 - 3 } & $40^{\circ} \mathrm{C}$ & 108 \\
\cline { 2 - 3 } & $-40^{\circ} \mathrm{C}$ & 80,500 \\
\hline Viscosity Index & 137 \\
\hline Flash Point & $300^{\circ} \mathrm{C}$ \\
\hline Vapor Pressure & $25^{\circ} \mathrm{C}, 3.999 \times 10^{-9} \mathrm{~Pa}$ \\
\hline Evaporation & $100^{\circ} \mathrm{C}, 24 \mathrm{~h}$, none \\
\hline
\end{tabular}

In order to study the chemical composition of the wear scar area and the tribochemical changes in the lubricant film, micro-spot XPS was employed. The XPS analysis was performed by irradiating the surface with a beam of monochromatic $\mathrm{Al} \mathrm{K \alpha}(1486.6 \mathrm{eV})$ $\mathrm{X}$-Ray. The diameter of the analysis area was $120 \mu \mathrm{m}$. The binding energy reference was taken as the main component of the $\mathrm{C} 1 \mathrm{~s}$ peak at $284.5 \mathrm{eV}$ for adventitious carbon.

\section{Results and discussion}

3.1. Desorption of gaseous products during sliding process

The surface of the steel disk was covered with a layer composed of metal oxides, chemisorbed water and organic contaminants, which were less active than nascent surface. In order to obtain the nascent surface,
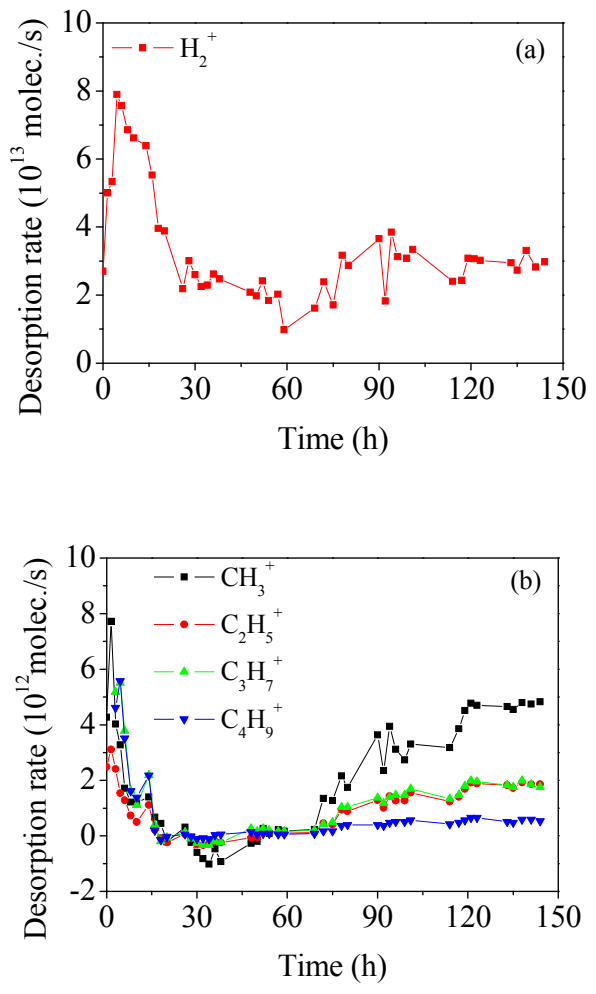

Fig.3 Desorption of products during surface contamination removals (Sliding velocity- $0.02 \mathrm{~m} / \mathrm{s}$, Load-8 $\mathrm{N}$ )

(a) Hydrogen (b) Gaseous hydrocarbons

the layers should be removed. Figure 3a shows desorption of hydrogen during sliding experiment. First desorption rate of hydrogen decreased when the sliding was continued for 30 hours. After about 75 hours, it increased gradually and then became stable. Figure $3 \mathrm{~b}$ shows desorption of gaseous hydrocarbons during the sliding experiment. Firstly desorption rate decreased and about 25 hours later no obvious gaseous hydrocarbons were observed. After 75 hours, desorption rate of gaseous hydrocarbons increase again and then gradually became stable until about 120 hours.

Hydrogen evolution at the initial stage did not originate from a catalytic decomposition of lubricant, but from the thermal decomposition of surface hydroxyl groups and chemisorbed water ${ }^{10)}$. Gaseous hydrocarbons at the initial stage also did not originate from catalytic decomposition of lubricant, but possibly from the decomposition of contaminants and di-tert-dodecyl disulfide. With removal of the contaminant, desorption of hydrogen and gaseous hydrocarbons decreased until about 75 hours. While contaminant and oxide layer were removed and the nascent surface with active sites were generated by friction for 75 hours, desorption of hydrogen and gaseous hydrocarbons increased and reached a constant value. Minus values for desorption rate of gaseous hydrocarbons as shown in Figure 3(b) maybe due to the experimental errors. 

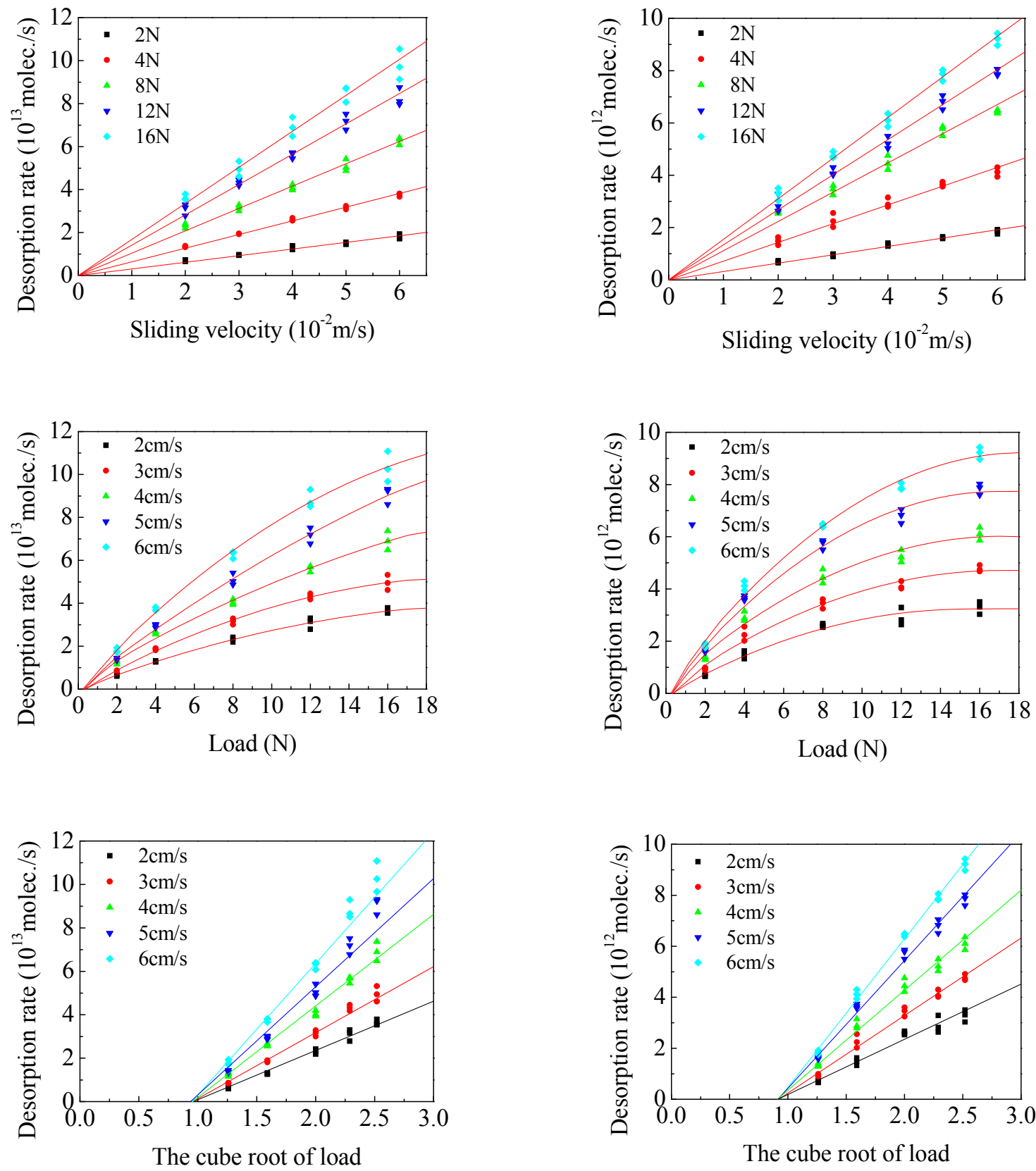

Fig.4 Dependence of desorption rate of hydrogen on sliding conditions

Compared with the results of additive-free MAC oil, in which hydrogen desorption decreased firstly and increased after only 7 hours ${ }^{5)}$, it took much longer time to increase hydrogen desorption by the addition of the sulfide additive. When additive was introduced, not only the contaminations and oxide layers, but also the chemisorbed layer of additive on oxide layer should be removed to obtain the nascent surface. Consequently, the additive prolonged the induction period of the decomposition.

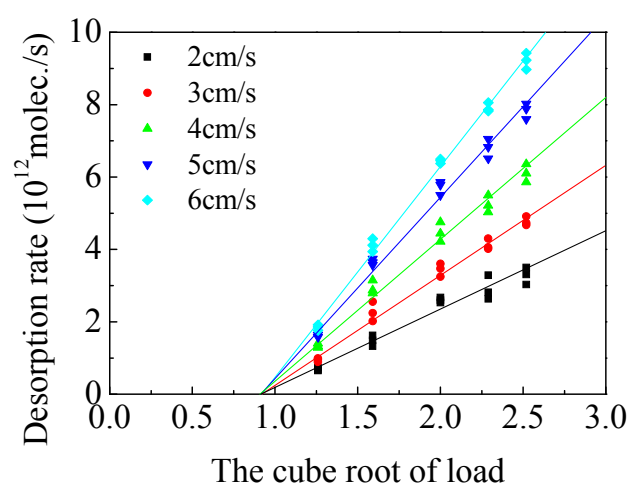

Fig.5 Dependence desorption rate of methane on sliding conditions

\subsection{Desorption rate of reaction products}

From the pressure change due to desorption, desorption rate $\left(R_{d}\right.$, molecules/s) of reaction products by mechanical activation can be estimated by the following equation $^{7,8)}$.

$$
R_{d}=C \Delta P / k T
$$

where, $C$ is a conductance at the gas outlet of the vacuum chamber; $\Delta P$ is pressure change due to 
desorption at the steady state and is proportional to the intensity change of the molecular and fragment ions; $k$ is Boltzmann's constant; and $T$ is absolute temperature.

Figure 4 shows the dependence of desorption rate of hydrogen on sliding conditions. It was found that desorption rate of hydrogen by mechanical activation increased proportionally with sliding velocity at any load tested in this study. On the other hand, desorption rate increased in a curvilinear relation with load. From the dependence of desorption rate of hydrogen on the cube root of load, it can be seen that all curves are linear and intercept with the axis of the cube root of load at the same point of $0.9 \mathrm{~N}$, which indicated that the decomposition was observed above this load. Therefore, this point is named as a critical load for the activation ${ }^{5}$.

The dependence of desorption rate of methane on sliding conditions is given in figure 5 . It can be seen that desorption rate of methane on nascent surface of bearing steel increased proportionally with sliding velocity, and curvilinearly with load. Desorption rate increased linearly with the cube root of load. The critical load for the activation is also observed at load of $0.9 \mathrm{~N}$. For other gaseous hydrocarbons, such as ethane, propane, and butane, similar results were observed. The mechanism of the dependence of desoprtion rate on mechanical conditions has been discussed in detail elsewhere $^{5)}$.

Compared with the results of additive-free MAC oil ${ }^{5)}$,
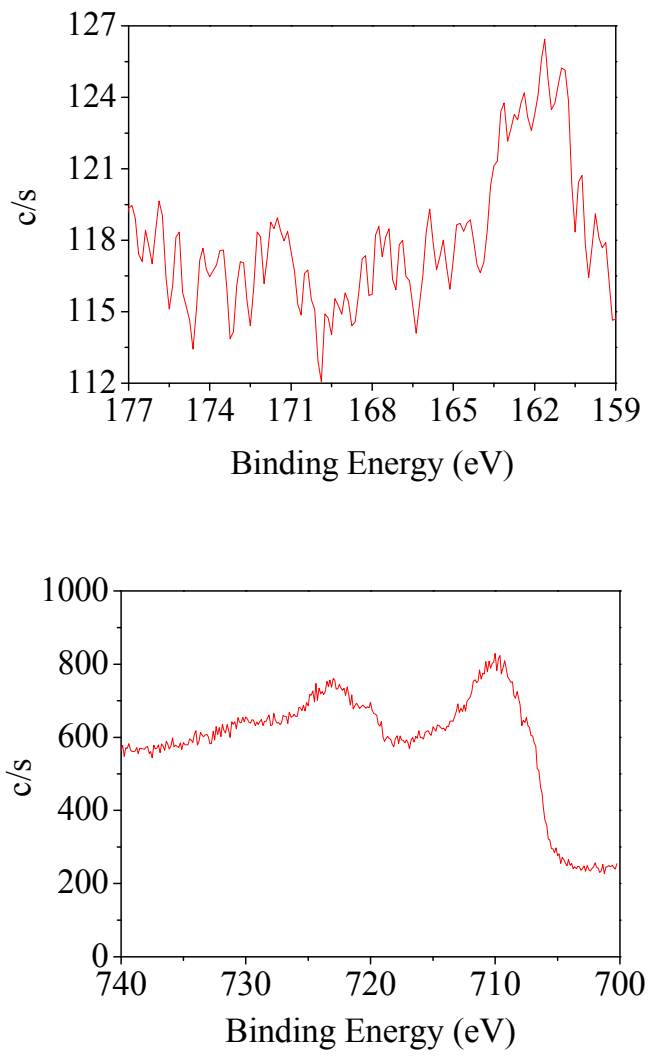

Fig. 6 XPS spectra of iron and sulfide collected from the wear scar for example desorption rate of methane generated from additive-free MAC oil was $7.4 \times 10^{12} \mathrm{molec} . / \mathrm{s}$ at the load of $8 \mathrm{~N}$ and sliding velocity of $4 \mathrm{~cm} / \mathrm{s}$, desorption rates of gaseous hydrocarbons generated from MAC oil with additive decreased obviously, which indicated the additive was effective to prevent the decomposition of MAC oil. In fact, when additive concentration was added to $2 \mathrm{wt} \%$, desorption rate of methane decreased to $1.8 \times 10^{12} \mathrm{molec} . / \mathrm{s}$ at the load of $8 \mathrm{~N}$ and sliding velocity of $4 \mathrm{~cm} / \mathrm{s}$.

\subsection{XPS analysis of the wear scar surface}

Figure 6 shows the XPS spectra of iron and sulfide collected from the wear scar lubricated by MAC oil with $1 \%$ additive. The signal of the $S(2 p)$ at the binding energy of $161.6 \mathrm{eV}$ suggests that FeS was formed on the wear scar. The Fe (2p 3/2) signal located at the binding energy of $707.2 \mathrm{eV}$ relates to metallic Fe and the main binding energy of 709.3 710.9 eV relates to $\mathrm{FeO}$ and $\mathrm{Fe}_{2} \mathrm{O}_{3}$ respectively. Formation of $\mathrm{FeS}$ is also supported by the signal of Fe (2p 3/2) located at the binding energy of $712.7 \mathrm{eV}^{11)}$.

3.4. Decomposition mechanism of MAC oil on bearing steel surface

The ratio of desorption rate of different ions represents desorption mechanism because distribution of products may be uniform in a certain decomposition mechanism. Fig. 7 shows the dependence of the ratio of
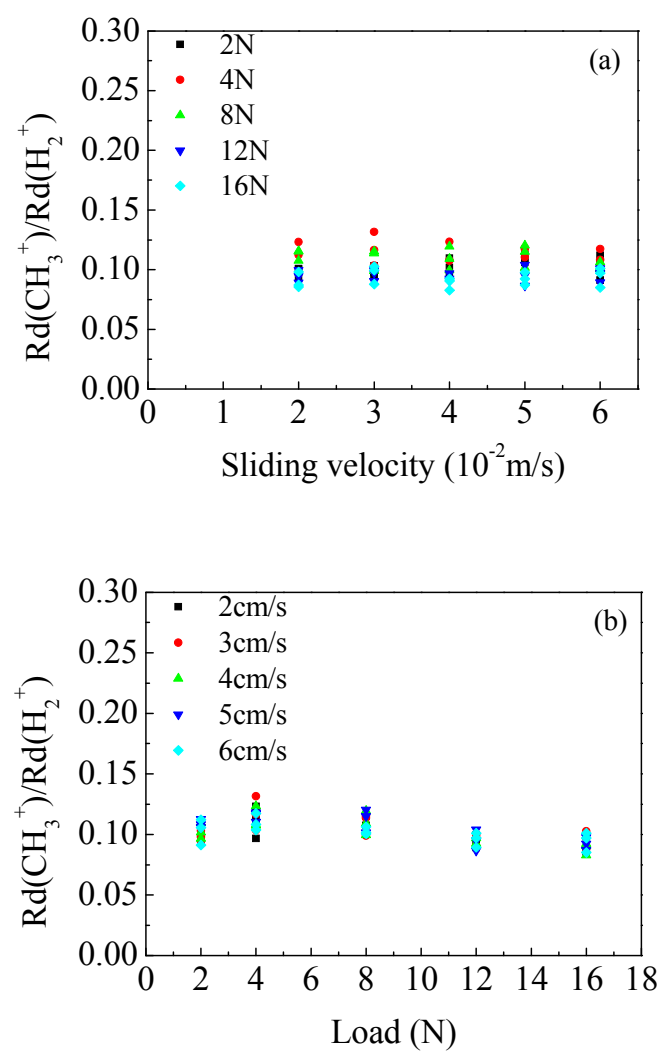

Fig. 7 Dependence of ratio of desorption rate of methane to hydrogen on mechanical conditions 
desorption rate of methane to hydrogen on mechanical conditions. It is found that the ratio of desorption rate of methane to hydrogen was not dependent on sliding velocity and load in this study. For other gaseous hydrocarbons, similar results were obtained. This means desorption mechanism of MAC oil with sulfide additive at mechanical contact of steel was not affected by sliding conditions tested in this study.

From above results, a possible process of tribochemical reaction and decomposition of MAC oil with additive on the nascent surface of bearing steel is proposed as followed. Firstly, active sites on the nascent surface are generated by scratch. It is expected that defects are generated on nascent surfaces by mechanical contacts. It is well-known that surface defects act as an active site for certain reactions ${ }^{12}$. Secondly, additive molecules and lubricant molecules competitively chemisorb on these active sites. Hydrogen and gaseous hydrocarbon desorbed as the tribochemical products. Additive molecules react with nascent surface by sulfide-iron bond and suppress the catalytic activity of active sites. As a result, decomposition of MAC oil with sulfur-containing additive decreased obviously compared with additive-free MAC oil.

\section{Conclusions}

Effect of di-tert-dodecyl disulfide as an additive on tribochemical decomposition of MAC oil on the nascent surface of bearing steel 52100 was investigated.

The additive prolonged the induction period of decomposition. After the removal of surface layers such as metal oxides and contaminants during the rubbing process, active sites were generated, on which additive molecules and lubricant molecules competitively absorbed. Hydrogen and gaseous hydrocarbons desorbed as tribochemical reaction products. The desorption rate of hydrogen and gaseous hydrocarbons increased proportionally with sliding velocity, and linearly with the cube root of load. Desorption mechanism was not affected by sliding conditions in this study. Additive molecules react with nascent surface by sulfide-iron bond and suppress the catalytic activity of active sites. As a result, decomposition of MAC oil decreased.

\section{Reference}

[1] Venier, C. G. and Casserly, E. W., "Multiply-Alkylated Cyclopentanes(MACs): A
New Class of Synthesized Hydrocarbon Fluids," Lubri. Engr., 47, 7, 1991, 586-591.

[2] John, P. J., Cutler, J. N. and Sanders, J. H., "Tribological Behavior of a Multialkylated Cyclopentane Oil under Ultrahigh Vacuum Conditions," Tribol. Lett., 9, 3-4, 2000, 167-173.

[3] Jansen, M. J., Jones, W. R. and Pepper, S. V., "Evaluation of an In-Situ, Liquid Lubrication System for Space Mechanisms Using a Vacuum Spiral Orbit Tribometer," NASA Glenn Research Center Technical Reports, NASA/TM-2002-211683.

[4] Marchetti, M., Jones, W. R., Street, K. W., Wheeler, D., Dixon, D., Jansen, M. J. and Kimura, H., "Tribological Performance of Some Pennzane ${ }^{\circledR}$-Based Greases for Vacuum Applications,” Tribol. Lett., 12, 4, 2002, 209-216.

[5] Lu, R., Minami, I., Nanao, H. and Mori, S., "Investigation of Decomposition of Hydrocarbon Oil on the Nascent Surface of Steel," Tribol. Lett., 27, 1, 2007, 25-30.

[6] Kohara, M., Kawamura, T. and Egami, M., "Study on Mechanism of Hydrogen Generation from Lubricants," Tribol. Trans., 49, 1, 2006, 53-60.

[7] Mori, S., Suginoya, M. and Tamai, Y., "Chemisorption of Organic Compounds on a Clean Aluminum Surface Prepared by Cutting under High Vacuum," ASLE Trans., 25, 2, 1982, 261-266.

[8] Mori, S., "Adsorption of Benzene on the Fresh Steel Surface Formed by Cutting under High Vacuum,” Appl. Surf. Sci., 27, 4, 1987, 401-410.

[9] Wu, X., Cong, P., Nanao, H., Kobayashi, K. and Mori, S., "Chemisorption and Tribochemical Reaction Mechanisms of HFC-134a on Nascent Surfaces," Langmuir, 18, 26, 2002, 10122-10127.

[10] Mori, S. and Yoshida, M., "Decomposition of Aromatic Compounds on Cut Nickel Surface," STLE Trans., 31, 1, 1988, 128-132.

[11] Wagner, C. D., Riggs, W. M., Davis, L. E., Moulder, J. F. and Muilenberg, G. E., "Handbook of X-Ray Photoelectron Spectroscopy," Perkin Elmer Corporation, Eden Prairie, Minnesota, 1979.

[12] Somorjai, G. A., "Introduction to Surface Chemistry and Catalysis," Wiley-Interscience, New York, 1994. 